\title{
IMPACTO DA DOR NA VIDA DE PORTADORES DE DISFUNÇÃO TEMPOROMANDIBULAR
}

\author{
PAIN IMPACT ON LIFE OF PATIENTS WITH \\ TEMPOROMANDIBULAR DISORDER
}

\begin{abstract}
Anamaria Siriani de OLIVEIRA
Fisioterapeuta, Doutora em Biologia Buco-Dental - Faculdade de Medicina de Ribeirão Preto - FMRP-USP.

Camila Colombo BERMUDEZ

Rodrigo Alves de SOUZA

Carina Maria Freitas SOUZA

Fisioterapeutas - Centro Universitário de Araraquara, UNIARA, Araraquara, São Paulo, Brasil.

\section{Elton Matias DIAS}

Mestrando em Fisioterapia - Centro Universitário do Triângulo, UNIT, Uberlândia, Minas Gerais, Brasil.

Carlos Eduardo dos Santos CASTRO

Mestre em Fisioterapia - Universidade Federal de São Carlos, UFSCar, São Carlos, São Paulo, Brasil.

Fausto BÉRZIN

Cirurgião Dentista, Professor Titular de Anatomia - Faculdade de Odontologia de Piracicaba, FOP-UNICAMP, Piracicaba, São Paulo, Brasil.

Instituição onde foi realizado o trabalho: Centro Universitário de Araraquara, UNIARA, Araraquara, São Paulo, Brasil, Rua Carlos Gomes, 1338 - Centro, CEP 14801-340.
\end{abstract} \begin{abstract}
$\mathcal{L}_{\text {oi realizado um estudo do impacto da dor na vida de indivíduos portadores de disfunção da articulação }}$ temporomandibular (DTM). Foram estudados 22 pacientes (20 mulheres e dois homens, com idade média de 28 anos) portadores de DTM que procuraram atendimento fisioterapêutico especializado, submetidos a uma versão brasileira do Questionário McGill de Dor (Br-MPQ), que inclui questões específicas sobre a qualidade de vida, como: 1) prejuízo social; 2) atividades da vida diária; 3) percepção do outro; 4) tolerância à dor; 5) sensação de estar doente; 6) sensação de utilidade; 7) satisfação com a vida. Os resultados mostraram que a dor da DTM prejudicou as atividades do trabalho (59,09\%), da escola $(59,09 \%)$, o sono $(68,18 \%)$ e o apetite/alimentação (63,64\%). Os autores concluíram que a dor da DTM tem um impacto negativo na qualidade de vida do paciente e que o questionário empregado, embora não específico, permitiu avalia-lo adequadamente.
\end{abstract}

UNITERMOS: Dor; Qualidade de vida; Questionário McGill de Dor; Disfunção temporomandibular.

\section{INTRODUÇÃO}

Na disfunção temporomandibular (DTM) as condições musculoesqueléticas, quer da região cervical, quer da musculatura da mastigação, são a maior causa de dor não dental na região orofacial ${ }^{12}$. Por sua origem ser multifatorial, muitos estudos buscam definir os mecanismos predisponentes, causais e perpetuantes desta patologia. Entretanto, a avaliação e descrição da dor relacionada à disfunção da articulação temporomandibular precisam ser melhores esclarecidas.
A dor é definida, segundo a Internacional Association for the Study of Pain (IASP), como uma experiência sensorial e emocional desagradável, associada a um dano tissular real ou potencial ou descrita em termos de tal dano ${ }^{14}$. Nos termos dessa definição, não apenas os componentes físico e químico envolvidos no evento doloroso são considerados, mas também os aspectos subjetivo e psicológico, que são cruciais na compreensão da queixa dolorosa, especialmente, em pacientes com dor crônica.

A inclusão de aspectos afetivo-emocionais 
subjetivos, na definição de dor adotada nesta pesquisa, exigiu a aplicação de uma ferramenta de avaliação multidimensional: o questionário de dor McGill (MPQ). Na versão brasileira deste questionário (Br-MPQ), proposta por Castro $^{3}$, o impacto da dor na vida do paciente é avaliado por questões relacionadas ao prejuízo social, desenvolvimento das atividades da vida diária e percepção do paciente sobre a reação de terceiros a sua condição dolorosa.

Reisine et al. ${ }^{15}$ estudaram a utilidade da aplicação de indicadores padronizados, incluindo o questionário McGill, para medir o impacto da dor orofacial na qualidade de vida de 152 pacientes. Entre as condições estudadas, 48 pacientes apresentavam DTM, 33 doenças periodontais, 23 próteses e 48 pacientes em situação de retorno clínico por diversas condições. Os três primeiros grupos de pacientes relataram diferentes impactos na qualidade de vida e o impacto foi, particularmente, severo para os portadores de DTM. Os indicadores utilizados foram sensíveis para diferenciar entre os grupos de pacientes e foram considerados promissores no acompanhamento da qualidade de vida e na coleta de dados epidemiológicos.

Embora existam evidências para acreditar que as DTM e outras condições dolorosas da face causem algum impacto na qualidade de vida ${ }^{5}$, um pequeno número de estudos documenta o uso de questionários específicos ou mesmo de ferramentas multidimensionais com este intuito. Assim, o objetivo deste trabalho é descrever os relatos subjetivos de portadores de DTM sobre o impacto da dor em suas vidas, através das questões propostas na versão brasileira do questionário $\mathrm{MPQ}^{3}$.

\section{MATERIAL E MÉTODOS}

\section{Voluntários}

Para realização deste trabalho foi selecionado um grupo de 22 pacientes, sendo 20 mulheres e 2 homens, com faixa etária entre 17 a 55 anos de idade (28 \pm 11 anos) da cidade de Araraquara e região, que procuraram atendimento fisioterapêutico, com diagnóstico odontológico de DTM. Todos os voluntários apresentavam história de dores decorrentes da DTM há pelo menos seis meses, e com mais de uma tentativa de tratamento sem sucesso que, segundo a IASP $(1994)^{10}$, caracteriza um clínico de dor crônica. A todos os voluntários foi solicitado o preenchimento do consentimento formal de participação após o completo esclarecimento dos objetivos da pesquisa.

\section{Procedimento}

A avaliação do impacto da dor na vida dos pacientes foi realizada por meio das respostas às perguntas específicas do questionário de dor $\mathrm{Br}-\mathrm{MPQ}^{3}$. As instruções necessárias para o preenchimento do questionário foram dadas até que o voluntário as tivesse compreendido completamente. O preenchimento foi realizado pelo voluntário, enquanto o terapeuta permaneceu à disposição para qualquer esclarecimento.

Neste questionário o paciente indicou o impacto da dor sobre sua vida em subitens das categorias: (1) prejuízo social, (2) atividades da vida diária e (3) percepção do outro, conforme ilustrado na parte esquerda da Figura 1. As possíveis respostas, para cada um dos itens em questão, eram assinaladas pela associação de uma escala ordinal/nominal conforme apresentado no canto superior esquerdo da Figura 1. Também foram incluídas as questões sobre (4) tolerância à dor, (5) sensação de estar doente, (6) sensação de utilidade e (7) satisfação com a vida, como apresentadas no lado direito da Figura 1.

Deixou-se claro que poderia ser escolhido somente um número ou afirmativa em cada um dos subitens. $\mathrm{O}$ questionário foi aplicado sem controle de tempo para o completo preenchimento, a fim de que não houvesse razões de conduzir o voluntário a respostas apressadas. Nenhum dos voluntários demonstrou dificuldade em seguir as instruções dadas, nem para entender o significado das questões, palavras ou expressões. Em média o tempo gasto com a aplicação do questionário completo foi de 20 minutos.

\section{Análise dos Dados}

Do $\mathrm{Br}-\mathrm{MPQ}^{3}$ são analisados os achados relativos ao impacto da dor na vida do paciente nos subitens das categorias: (1) prejuízo social, (2) atividades da vida diária e (3) percepção do outro, e nas questões sobre (4) tolerância à dor, (5) sensação de estar doente, (6) sensação de utilidade e (7) satisfação com a vida. A análise descritiva escolhida foi a distribuição de freqüência dos dados obtidos para cada um dos subitens avaliados. O teste qui-quadrado foi aplicado aos valores absolutos para evidenciar diferenças entre as respostas positivas ao impacto e respostas negativas atribuídas às variáveis estudadas, com nível de significância de 5\%. 
Para indicar o quanto a dor influencia em seu cotidiano, use a seguinte numeração: (1) não; (2) pouco; (3) mais ou menos; (4) muito; (5) totalmente/sempre. As alternativas recuadas serão apenas assinaladas em caso afirmativo.

(As alternativas recuadas serão apenas assinaladas)

Prejuízo Social: a dor afeta

( ) no trabalho

( ) perda de dias de trabalho

( ) licença saúde

( ) perda de emprego

( ) aposentadoria

( ) nas atividades escolares

( ) no lazer

( ) nas atividades domiciliares

( ) no relacionamento familiar

( ) no relacionamento com os amigos

Atividades da vida diária

( ) sono

( ) insônia inicial

( ) insônia terminal

( ) sono não reparador

( ) o apetite/alimentação

( ) a higiene pessoal

( ) vestir-se

( ) a locomoção

Percepção do Outro: As pessoas

( ) ficam irritadas comigo

( ) expressam frustração

( ) sentem raiva de mim

( ) me ignoram

FIGURA 1- Questões sobre o impacto da dor na vida do paciente. Parte integrante da versão brasileira do questionário McGill de dor, proposta por Castro (1999)

\section{RESULTADOS}

Os achados referentes à percepção dos pacientes sobre o impacto da dor em aspectos sociais de suas vidas são apresentados na Tabela 1. Os resultados mostram que 59,09\% dos pacientes referem algum grau de prejuízo no trabalho e nas atividades escolares; $50 \%$, nas atividades de lazer e no relacionamento familiar; 54,55\% nas atividades domiciliares. No entanto, todas as variáveis acima citadas não apresentaram diferenças estatisticamente significativas quando comparadas com o número de respostas negativas a estes impactos ( $\mathrm{p}>0,05)$. O prejuízo no relacionamento com os amigos foi de $27,27 \%$ e quando o número de respostas positivas foi comparado ao de
Assinale a alternativa que mais se identifica com você:

Tolerar a dor:

( ) não é difícil

( ) é um pouco difícil

( ) é difícil

( ) é muito difícil

( ) é impossível

Você se sente doente?

( ) não

( ) um pouco

( ) muito

( ) totalmente

Você se sente útil?

( ) sim

( ) menos que antes

( ) inútil

( ) muito inútil

( ) totalmente inútil

Sua vida é satisfatória

( ) $\operatorname{sim}$

( ) em parte

( ) insatisfatória

( ) completamente insatisfatória respostas negativas, o teste qui-quadrado mostrou que a dor não provoca impacto nesta variável $(\mathrm{p}>0,05)$.

A Tabela 1 mostra também os achados do impacto da dor nas atividades da vida diária dos pacientes estudados. Os subitens sono $(68,18 \%)$ e apetite/ alimentação $(63,64 \%)$ foram os que exibiram maiores porcentagens de respostas relativas a algum grau de prejuízo ( $>00,05)$. A higiene pessoal foi indicada como prejudicada pela dor em $22,73 \%$ dos pacientes, enquanto a locomoção e o ato de vestir-se foram apontados por apenas 9,09\% dos pacientes. Estas três variáveis não foram influenciadas pela dor ( $p>0,05)$.

A percepção que o portador de DTM tem sobre o comportamento das outras pessoas em relação a sua dor (Tabela 1) mostrou que 31,82\% deles acreditam 
que as pessoas ficam irritadas ( $p>0,05), 18,18 \%$ sentem frustração $(p>0,05)$ e $4,55 \%$ o ignoram ( $>0.05)$.

Os resultados apresentados na Tabela 1 também mostram que $77,27 \%$ dos portadores de DTM têm alguma dificuldade de tolerar a dor $(\mathrm{p}<0,05)$. Porém, apenas $36,36 \%$ sentem-se um pouco doentes. Do total da amostra, 27,27\% acreditam que são menos úteis que antes da instalação da patologia e 22,73\% acham que sua vida não é completamente satisfatória devido à dor. Estas três variáveis não foram influenciadas pela dor $(\mathrm{p}>0,05)$.

\section{DISCUSSÃO}

Existe um consenso sobre o fato de dores crônicas produzirem efeitos não só biológicos, mas também psicológicos e sociais ${ }^{1}$, que merecem atenção na avaliação ${ }^{8}$ e que, muitas vezes, são controlados ou revertidos por medidas terapêuticas ${ }^{2}$.

Os resultados dessa pesquisa indicaram que, nas perguntas específicas para avaliar o impacto da dor na vida dos portadores de DTM com quadro crônico, o trabalho, as atividades escolares, o sono e o apetite/ alimentação foram as categorias mais influenciadas pelo quadro doloroso, embora nem todas tenham apresentado diferenças significativas quando comparadas às repostas negativas ao impacto.

A dificuldade de mastigação e até mesmo a recusa desta atividade foi anteriormente relatada como uma preocupação para o estado nutricional de crianças com DTM artrogênica decorrente da artrite reumatóide ${ }^{9}$ e estudos tentam correlacionar a diminuição da eficiência mastigatória com a severidade da disfunção ${ }^{6}$.

Utilizando uma ferramenta específica, o Oral Health Impact Profile, Murray, et al. ${ }^{13}$ avaliaram 121 pacientes em sua primeira visita a uma unidade especializada na pesquisa da dor craniofacial, a fim de mensurar a qualidade de vida destes pacientes e encontraram resultados semelhantes ao do presente estudo. Os achados dos autores supracitados mostraram um significante impacto na qualidade de vida dos portadores de DTM quando comparados à população sem queixa de dor. Os relatos de problemas funcionais, como dificuldade para mastigar determinados alimentos,

TABELA 1- Distribuição de freqüência das respostas, dos portadores de disfunção temporomandibular, para os subitens de avaliação do impacto da dor e seu prejuízo social, nas atividades da vida diária e na percepção que o paciente tem sobre o comportamento das pessoas. Valores em porcentagem $(n=22)$

\begin{tabular}{|c|c|c|c|c|c|}
\hline Prejuízo Social: a dor afeta & $\begin{array}{l}\text { Não } \\
(\%)\end{array}$ & $\begin{array}{l}\text { Um pouco } \\
(\%)\end{array}$ & $\begin{array}{c}\text { Mais ou menos } \\
\text { (\%) }\end{array}$ & $\begin{array}{c}\text { Muito } \\
(\%)\end{array}$ & $\begin{array}{c}\text { Totalmente/ Sempre } \\
(\%)\end{array}$ \\
\hline No trabalho & 40,91 & 40,91 & 0,00 & 18,18 & 0,00 \\
\hline Nas atividades escolares & 40,91 & 36,36 & 9,09 & 13,64 & 0,00 \\
\hline No lazer & 50,00 & 27,27 & 13,64 & 9,09 & 0,00 \\
\hline Nas atividades domiciliares & 45,45 & 31,82 & 9,09 & 9,09 & 4,55 \\
\hline No relacionamento familiar & 50,00 & 22,73 & 4,55 & 18,18 & 4,55 \\
\hline No relacionamento com os amigos & 72,73 & 9,09 & 9,09 & 9,09 & 0,00 \\
\hline \multicolumn{6}{|l|}{$\begin{array}{l}\text { Atividades da Vida Diária: } \\
\text { a dor afeta }\end{array}$} \\
\hline Sono & 31,82 & 27,27 & 13,64 & 27,27 & 0,00 \\
\hline Apetite/Alimentação & 36,36 & 22,73 & 22,73 & 9,09 & 9,09 \\
\hline Higiena pessoal & 77,27 & 9,09 & 9,09 & 4,55 & 0,00 \\
\hline Vestir-se & 90,91 & 0,00 & 9,09 & 0,00 & 0,00 \\
\hline Locomoção & 90,91 & 4,55 & 4,55 & 0,00 & 0,00 \\
\hline \multicolumn{6}{|l|}{ Percepção do outro: } \\
\hline \multicolumn{6}{|l|}{ As pessoas } \\
\hline Ficam Irritadas comigo & 68,18 & 9,09 & 13,64 & 4,55 & 4,55 \\
\hline Expressam frustação & 81,82 & 4,55 & 9,09 & 4,55 & 0,00 \\
\hline Sentem raiva de mim & 100,00 & 0,00 & 0,00 & 0,00 & 0,00 \\
\hline Me ignoram & 95,45 & 4,55 & 0,00 & 0,00 & 0,00 \\
\hline
\end{tabular}


foram quatro vezes maiores para os pacientes estudados, e os relatos de depressão nove vezes maior.

A qualidade do sono, apontada como prejudicada por mais da metade dos voluntários deste estudo, foi anteriormente relacionada à percepção e à severidade da dor em pacientes com DTM ${ }^{16}$. Os resultados do citado estudo apontaram que os pacientes, que relatavam pobre qualidade do sono, tinham os índices de intensidade de dor mais elevados e menor percepção de controle sobre suas vidas.

O comprometimento das atividades do trabalho e atividades escolares também foi apontado por mais da metade dos voluntários deste estudo. No entanto, nenhum dos pacientes apontou licença ou falta/ausência no trabalho devido a sua dor. Macfarlane et al. ${ }^{11}$ demonstraram, em 2504 pacientes, um importante impacto da dor orofacial no trabalho, com $17 \%$ de relatos de perda de dias de trabalho e incapacidade de desenvolver suas atividades como antes da dor. No entanto, a amostra do citado estudo inclui pacientes com dores orofaciais de diferentes origens, incluindo as odontogênicas que, por suas características de quadro agudo, invalidam uma correlação direta dos achados dos autores com os do presente estudo.

Embora a intolerância à dor tenha sido um achado freqüente, o descritor mais escolhido foi "tolerar a dor é um pouco difícil”. Este resultado relaciona-se ao fato dos pacientes com dor crônica apresentarem, em geral, intensidades menores de dor, estados psicológicos depressivos e aceitação/resignação ${ }^{4}$.

A sensação de insatisfação com a vida e diminuição do nível de atividade apresentados neste estudo acometeram um número reduzido de pacientes. Achados semelhantes, como a limitação da função social, do bem estar emocional e do nível de energia, foram relatados por Di Fabio ${ }^{7}$ em pacientes com DTM. Diferente do achado do presente estudo, o autor supracitado relacionou estes achados ao quadro crônico de instalação da disfunção.

Embora a versão brasileira do questionário McGill de dor, proposta por Castro ${ }^{3}$, seja uma ferramenta multidimensional de avaliação de dor e, portanto, não específica para mensurar qualidade de vida relacionada a problemas orofaciais, a concordância de achados deste estudo com os de estudos específicos anteriormente realizados, permite sugerir a utilização deste para quantificar e acompanhar a evolução da dor envolvida na DTM e seu impacto na vida do paciente. No entanto, o uso de ferramentas específicas deve ser considerado sempre que uma avaliação aprofundada for desejada e mais estudos devem avaliar um número maior de sujeitos para confirmar os resultados aqui apresentados.

\section{CONCLUSÃO}

Nestas condições experimentais, é possível concluir que portadores de DTM crônica apresentam algum grau de impacto da dor em suas vidas, especialmente nas atividades do trabalho, da escola, no sono e no apetite/alimentação. Embora não específica para avaliação da qualidade de vida, a versão brasileira do questionário McGill, proposta por Castro ${ }^{3}$ foi capaz de revelar este impacto. Um número maior de sujeitos deve ser avaliado pela mesma ferramenta para confirmar os resultados aqui apresentados.

\section{AGRADECIMENTOS}

Ao Conselho Nacional de Desenvolvimento Científico e Tecnológico - CNPq pela bolsa de doutorado referente ao processo $n^{\circ} 140360 / 2000-0$.

\section{ABSTRACT}

A study on the impact of pain in patients with temporomandibular joint disorder (TMD) was carried out. Twenty two patients (20 women, two men, 28 years of age on average) with TMD who looked for specialized physiotherapy care were submitted to a brazilian version of McGill Pain Questionnaire (BrMPQ), which includes questions about life quality, as follows: 1) social loss; 2) daily life activities; 3) third party's perception; 4) pain tolerance; 5) sensation of being sick; 6) sensation of being useless; 7) life satisfaction. The results showed that pain related to the TMD significantly affected work activities (59.09\%), school activities (59.09\%), sleeping (68.18\%) and appetite/feeding (63.64\%). The authors conclude that pain from TMD has a negative impact on the patients' life quality and that, although unspecific, the questionnaire used allowed for an adequate evaluation of the impact.

UNITERMS: Pain; Life quality; McGill pain questionnarie; Temporomandibular disorder. 


\section{REFERÊNCIAS}

1- Brown FF, Robinson ME, Riley JL, Gremillion HA. Pain severity, negative affect, and microstressers as predictors of life interference in TMD patients. Cranio 1996 Jan; 14(1):63-70.

2- Carlson CR, Bertrand PM, Ehrlich AD, Maxwell AW, Burton RG. Physical self-regulation training for the management of temporomandibular disorders. J Orofac Pain. 2001 Winter; 15(1):47-55.

3- Castro CES. A formulação lingüística da dor - versão brasileira do questionário McGill de Dor. São Carlos; 1999. [Dissertação de Mestrado - Universidade Federal de São Carlos].

4- Cohen MJ, McArthur DL, Vulpe M, Schandler SL, Gerber KE. Comparing chronic pain from spinal cord injury to chronic pain of other origins. Pain 1988 Oct; 35(1): 57-63.

5- Dahlstrom L, Widmark G, Carlsson SG. Cognitive-behavioral profiles among different categories of orofacial pain patients: diagnostic and treatment implications. Eur J Oral Sci. 1997 Oct; 105(5 Pt 1):377-83.

6- Dao TT, Lund JP, Lavigne GJ. Comparison of pain and quality of life in bruxers and patients with myofascial pain of the masticatory muscles. J Orofac Pain 1994; 8(4): 350-6.

7- Di Fabio RP. Physical therapy for patients with TMD: a descriptive study of treatment, disability, and health status. J Orofac Pain 1998 Spring; 12(2):124-35.

8- Greene CS. The etiology of temporomandibular disorders: implications for treatment. J Orofac Pain. 2001 Spring; 15(2):93105.

9- Harper RP, Brown CM, Triplett MM, Villasenor A, Gatchel RJ. Masticatory function in patients with juvenile rheumatoid arthritis. Pediatr Dent 2000 May/Jun; 22(3): 200-6.

10- IASP. Classification of chronic pain: descriptors of chronic pain syndromes and definitions of pain terms. $2^{\text {th }}$ ed. Seatle: IASP Press; 1994.

11- Macfarlane TV, Blinkhorn AS, Davies RM, Kincey J, Worthington HV. Oro-facial pain in the community: prevalence and associated impact. Community Dent Oral Epidemiol 2002 Feb; 30(1): 52-60.

12- Magnusson T, Egermark I; Carlsson GE. A longitudinal epidemiologic study of signs and symptoms of temporomandibular disorders from 15 to 35 years of age. J Orofac Pain 2000 14(4): 310-9.

13- Murray GM, Phanachet I, Uchida S, Whittle T. The role of the human lateral pterygoid muscle in the control of horizontal jaw movements. J Orofac Pain 2001 15(4): 279-92.

14- PAIN. Classification of chronic pain syndromes and definitions of pain terms [S 217]. Pain 1986 Supplement 3.

15- Reisine ST, Fertig J, Weber J, Leder S. Impact of dental conditions on patients' quality of life. Community Dent Oral Epidemiol 1989; 17(1): 7-10.
16- Yatani H, Studts J, Cordova M, Carlson CR, Okeson JP. Comparison of sleep quality and clinical and psychologic characteristics in patients with temporomandibular disorders. J Orofac Pain 2002; 16(3): 221-8.

Recebido para publicação em: 18/12/2002

Aceito após reformulações em: 20/03/2003

Endereço para correspondência:

Anamaria Siriani de OLIVEIRA

Rua Adércia Machado Gontijo, 191 apto 14, Residencial

Flórida - Ribeirão Preto - SP

14026310, siriani@fmrp.usp.br, Fone: 016 602-2315 